This is a self-archived - parallel published version of this article in the publication archive of the University of Vaasa. It might differ from the original.

\title{
Developing a practical methodology to improve the health-care services: studying a neonatal intensive care unit case in Oman
}

Author(s): Al-Hinai, Nasr; Shamsuzzoha, Ahm

Title: Developing a practical methodology to improve the health-care services: studying a neonatal intensive care unit case in Oman

Year: $\quad 2020$

Version: Accepted manuscript

Copyright C2020 Emerald Publishing Limited. This manuscript version is made available under the Creative Commons Attribution-NonCommercial 4.o International (CC BY-NC 4.0) license, https://creativecommons.org/licenses/by-nc/4.o/

Please cite the original version:

Al-Hinai, N. \& Shamsuzzoha, A. (2020). Developing a practical methodology to improve the health-care services: studying a neonatal intensive care unit case in Oman. International Journal of Quality and Reliability Management. https://doi.org/10.1108/IJQRM-08-20200284 


\title{
Developing a practical methodology to improve the healthcare services: studying a neonatal intensive care unit case in Oman
}

\author{
Nasr Al Hinai \\ Department of Mechanical and Industrial Engineering, College of Engineering, \\ Sultan Qaboos University, Muscat, Oman, and \\ Ahm Shamsuzzoha \\ School of Technology and Innovations, University of Vaasa, Vaasa, Finland
}

\begin{abstract}
Purpose - This research study aims to develop a practical methodology to identify possible areas of improvements as well as exploring how to improve the healthcare staff flow within a selected department in a hospital.

Design/methodology/approach - It focuses on showing how to properly study and analyze the healthcare services and processes practiced at a selected department within a hospital. For this, several techniques like non-value adding activities, time motion study, spaghetti diagram, layout analysis, etc. are used.

Findings - To test the proposed methodology, a Neonatal Intensive Care Unit (NICU) of a hospital in Oman was considered as a case study. The study revealed that this unit has several potential improvements capabilities. Further, this study also discussed possible areas of improvements of this case unit and suggested how such improvements can be implemented.

Originality/value - Several possible improvements are suggested and are discussed with the hospital authority, which can be clarified as the re-layout of the NICU rooms, reorganization of the store to improve the staff flow, increase the work efficiency, introduction of HUSH policy, etc., which can enhance the entire operational system at the studied NICU.
\end{abstract}

Keywords Healthcare improvement, NICU, Six-sigma, Lean, Case study, Improvement Methodology, Oman

Paper type case study

\section{Introduction}

With the rapid developments in the field of healthcare, public and private hospitals are seeking to enhance their services and to improve their level of cares. There is no doubt that patient satisfaction is very important issue that needs a serious look (Benzies et al., 2019). However, the development of healthcare services must be viable with minimal cost such that hospital can run at an efficient way and avoid unnecessary increase in the already high healthcare costs. This indeed makes it a big challenge for hospitals and healthcare providers to satisfy these two competing objectives.

It is an absolute necessity to ensure that continuous healthcare is provided to a person when he/she needs it. While many may argue that this healthcare is mainly needed after a person grow elderly, this in fact is not true. Healthcare needs start from the early signs of life as a fetus (beginning of pregnancy). Pregnancy period is a very sensitive period and it requires a special and continuous care for both, the mother and the baby. It takes around 40 weeks for fetus to grow completely inside the mother's womb. In some special cases, it may be required to remove the baby from mother's womb before completing this period due to health concerns related to either 
the mother or her baby. Babies who are born before completing their pregnancy periods are referred to as premature babies (McGowan et al., 2017). Hospitals usually have a special unit dedicated to take care of those premature babies. Many hospitals refer to this unit as the Neonatal Intensive Care Unit (NICU). This unit is equipped with all the necessary equipment needed to take care of the premature babies. It has a specialized medical team consisting of physicians and nurses working in it. Their works start the moment the baby is removed from his/her mother's womb and it continues as long as it is needed.

Normally, newborn babies undergo go through numerous physical changes once they are outside of their mothers wombs. Leaving the womb implies that a child can no more rely upon his/her mother's body and placenta for critical physiological needs like getting the nutrients and transferring the wastes outside of their bodies. However, the completely grown baby's organs start performing these basic operations to satisfy these needs. On the other hand, premature babies may not be able to do so as some of their vital organs are still under development or not functioning as required. Here, NICU plays a vital and essential role during the growing process of the premature baby to monitor and to provide all necessary help during this period. Hence, the nature of the work in such unit is intensive and delicate. This makes it a challenging work environment as many processes and procedures have to be performed for each premature baby and many of these premature babies may require different attention than the others. From this point of view, this study is mainly focusing on proposing a practical methodology framework that can be followed and implemented to improve healthcare services.

To test the proposed methodology, it is applied to Neonatal Intensive Care Unit (NICU) in a local hospital in Oman. This case study aims to test the suggested methodology and to oversee how it can be used to study, analyze, define and suggest possible ways to improve the unit. Nevertheless, any suggested improvement to the unit has to be done under very restricted constraints of space, capacity and cost limitations. This study is therefore, emphasizes on how to use and apply methods and techniques such as lean and six sigma to increase the efficiency and improve the level of services at the NICU while meeting all the needs of the patients. This study is conducted after identifying and collecting the necessary data from the hospital, which were then carefully analyzed. Accordingly, from the data analysis several improvements and/or feasible solutions were proposed to improve the overall healthcare services at the NICU and to improve the staff flow within the unit. This study identified three main objectives, which are stated below:

1) To define proper approaches as necessary to investigate and to analyze the current operational processes at a healthcare unit.

2) To identify systematically possible problematic areas within the unit, where necessary improvements can be implemented.

3) To identify "non-value added" activities and to prioritize those activities to improve healthcare processes in the care unit.

All these three research objectives can be converted to three suitable research questions (RQs) that are answered within the scope of this research study. The three research questions are formulated as follows:

RQ 1: What are the effective processes to make the healthcare services more efficient?

RQ 2: What is the practical methodology to identify the bottlenecks in a healthcare unit for possible improvement?

RQ 3: How to identify the non-value added activities in a healthcare unit in order to prioritize its improvement? 
First research question is answered by reviewing the literatures and consulting with expert opinions, while second research question is answered by proposing proper methodology to identify the associated bottlenecks within the concerned NICU area. Third research question is answered by sorting out the non-value added activities with the objective to make necessary changes to enhance the operational activities at NICU.

The remaining of this article is structured as follows: Section 2 presents literature reviews related to the premature babies and neonatal outcomes along with implementation of lean and SixSigma in healthcare. Section 3 highlights the study methodology, while brief description of the case study is provided in Section 4. Necessary data collection and study results are analyzed along with suggested improvements for staff flow, stores and noise level reduction in Section 5, whereas, Section 6 outlines suggested improvements in the NICU. Finally, Section 7 concludes the main findings and summarizes the study.

\section{Literature review}

\subsection{Premature babies and the need for improvements}

Studies shows that babies born earlier than their complete pregnancy period usually have a higher risk of death during the first year (Patel et al., 2015). While a typical pregnancy endures 40 weeks, babies born before 37 weeks are considered as premature (Chalmers, 2017; Lakshmanan et al., 2017). Infants born between 34 and 36 weeks are termed somewhat mildly premature, while moderate prematurity is characterized as between 32 and 34 weeks. Babies delivered before 32 weeks are viewed as very premature (Webmd, 2020). Joseph et al. (2000) did a statistical study on the birth of American and Canadian babies. According to this study, babies who are born even a week earlier may face a fundamentally more danger of death in their first year than babies who are born in full term. The authors highlighted that it has been generally trusted that infants who might be termed moderately (intermediate) did not confront the health risks of very premature. However, the records concluded that premature babies who were born one to three weeks earlier had around three times higher chances of death in the first year of their life in the US in 1995, while in Canada from 1992 to 1994 it was four and half times higher. The results of the study should prompt doctors to keep a closer eye on infants born prematurely.

The NICU around the world achieved different developments levels by developing different means to solve the different challenges faced by these units. These developments resulted in providing better levels of care. Researchers studied, reviewed and discussed different methods to enhance the service performance and healthcare of NIC units around the world (Trajkovski et al., 2016; Jochen et al., 2018). Nevertheless, in the field of optimization, using mathematical approaches for modelling systems play an important role in understanding the system as well as how different parameters affect its overall performance. However, the overall hospital environment is an extremely complicated environment with many entities interacting with each other. Hence, attempting to develop a mathematical model describing a system of this nature becomes unrealistic approach. Therefore, approaches that treat the hospital units/wards/departments as a separate entity and then study and analyze them to explore how to improve them may ends up to be a more convenient approach. Along with this, many of the developed engineering concepts to improve the industrial sector proved to be very efficient to use in other fields, including the healthcare field. Indeed, many researchers concentrated on the successful implementation of applying lean and Six-Sigma concepts in healthcare to reduce waste and improve quality. 


\subsection{Reviews in neonatal outcomes}

The rapid developments in the care of premature babies and understanding of neonatal pathology and physiology improved dramatically the level of care for newborns. The EPICure study 2012 analyzed the survival rate to the age of 3 years of premature babies born at 22 to 26 weeks gestation in England (Moore et al., 2012). The data about premature babies has been collected and compared for the year 1995 and 2006. Authors follow-up those babies during their childhood stage. EPICure study signified that a $44 \%$ growing in the numbers of extremely premature babies being confess for neonatal care (Costeloe et al., 2009). The study likewise found that the proportion of babies who go out alive from the hospital rose for all premature babies born somewhere around 22 and 25 weeks increased from $40 \%$ in 1995 to $53 \%$ in 2006. Table 1 shows the proportion of babies born at each gestation who survived according to EPICure. It clearly confirms that the rates have risen significantly from 1995 to 2006 .

Table 1. Proportion of babies at each gestation who survived (Moore et al., 2012)

\begin{tabular}{|c|c|c|}
\hline Gestational age at birth & 1995 survival & 2006 survival \\
\hline 22 weeks & $1 \%$ & $7 \%$ \\
\hline 23 weeks & $11 \%$ & $22 \%$ \\
\hline 24 weeks & $26 \%$ & $42 \%$ \\
\hline 25 weeks & $44 \%$ & $66 \%$ \\
\hline
\end{tabular}

This increase in percentage of survivals is contributed to the medical team interventions through the NICU. In these units, nurses are an important key to provide care for the premature babies. They are responsible for looking after them and providing the necessary care for them all day around. Nursing consideration in a neonatal unit can be variable and complex. Usually, work of nurses is divided into three main working shifts, morning shift, evening shift and night shift. The level of the daily responsibilities depends on the baby's condition. Thus, the workload on any given day at NICU can fluctuate contingent upon work requirement. Accordingly, the workplace can be stressful in many days. One component that extraordinarily upgrades the work fulfillment is having good communication within the staff. This is considered as a core competency for the neonatal nurse. Besides, neonatal nurses report that 'group soul' on a neonatal unit where associates helped one another, can help them to remain working in neonatal consideration.

\subsection{Lean and Six-Sigma in healthcare}

According to many researches, hospitals around the world achieved good results in improving their services using lean and Six-Sigma concepts (Stelson et al., 2017; Henrique and Filho, 2020). Lean helps to reduce waste while Six-Sigma focuses on reducing the variation in the process or services (Costa et al., 2017; Improta et al., 2019). Until recently, many healthcare providers were operating on traditional way that ignored many important factors such as patient safety as well as the increasing healthcare cost (Antony et al., 2018; Henrique and Filho, 2020). Nevertheless, due to the increased pressure from governments, health organizations, patients and people needs, business requirements, etc. people and organization became more aware of the importance to focus on these factors. Factors that increases the safety aspects when providing healthcare services for the patients and the institution's staff members as well as economic factors related to increasing efficiency and reducing costs. Here comes the importance of applying the concepts of lean and Six-Sigma in 
different facilities (Butler et al., 2018; Sunder and Kunnath, 2020). These concepts help in providing the best care at the lowest possible costs. In practice, both concepts are applied to satisfy costumers' desires and needs.

Lean is the concept or the philosophy of being able to do more with less resource. It is about having the ability to eliminate the waste and to sustain after eliminating that waste (DelliFraine, 2010; Sunder et al., 2018). In addition, it targets to deliver superior performance while focusing on what customers want without creating problems, delays and errors. When considering healthcare, one side of lean may be related to organize the diagnosis and treatment processes over medical conditions when everyone else may take several months. In fact, lean is in general about reducing the waste and complexity in process and time. Nowadays, many healthcare providers are using lean as a tool to rethinking their processes. There are five basic principles that any organization should follow to implement lean. They understand the value, value stream, the flow, pull and observing the Kaizen principles (Peimbert-Garcia, 2019; Rodrigo, 2019). Lean can increase healthcare value delivery by improving healthcare quality and decreasing healthcare cost . There are many wastes that can be faced in healthcare industry, which can be eliminated by applying lean thinking or principles. Actually, much of the patient's time is spent in waiting. Hence, more than $80 \%$ of the time spent in a healthcare process is considered as waste.

Recently, the lean concept started to be more linked and associated with the concept of SixSigma. Six-Sigma is defined as a management philosophy, which is used to reduce defects in goods and services by measuring the quality, collecting data and analyzing result (Vaishnavi and Suresh, 2020; Peimbert-Garcia, 2019). Six-Sigma is based on a quality statistics that equates to 3.4 defects per million opportunities. This is the best performance level for any process. Six-Sigma has five steps process problem solving approach known as DMAIC (defines, measure, analyze, improve, control) (Pande, 2001). Six-Sigma approach was used to improve the quality and productivity for healthcare organizations, thus improving patient care. The successful story in applying Six-Sigma in Mount Caramel Health System in Columbus, USA was presented by Loay and DeYoung (2003). There were 7,300 employees and 1,200 physicians of medical staff in three hospitals system of Mount Carmel Health System in the spring of 2000 experiencing big financial challenges. After implementation of Six-Sigma Mount in Carmel Health System, several benefits were achieved such as a \$3.1 million of financial savings have been realized, employees and physicians satisfaction were improved and employees' retention rates were improved.

\subsection{Identified research gaps}

From the literature survey, it is noticed that many researches on the care of premature babies and understanding of neonatal pathology and physiology have been conducted. It is also noticed from literature survey that most of the studies conducted in these areas are conducted within the developed countries. However, very few studies are conducted on these areas in the developing countries, especially in the Middle-Eastern countries like Oman. Furthermore, a systematic and practical methodology that can be followed to improve health services by healthcare providers is still missing. In practice, the authors noted that when healthcare providers want to improve any of their services, they mostly appoint a third party consultant. Among the main challenges, facing such approach is ignoring the fact that people from different backgrounds and/or organizations needs time to build trust and form a performing team. To fulfill such research gap, the objective of this study is propose an innovative and practical methodology to improve healthcare services. The methodology aims to provide the team members with a clear path to enable them to better understand the current services, identify possible areas of improvements and accordingly 
recommended several improvement strategies to make this specific service area more effective and efficient. The methodology should consider how to foster the team members and help them to reduce the time needed to perform. Therefore, this study proposed an innovative methodology to support researchers to study the healthcare system, especially in neonatal unit in general. The proposed methodology was tested and implemented in a neonatal unit. Moreover, this study also focused to fulfill the research gap how to eliminate or minimize various wastes in neonatal intensive care unit by adopting lean and six sigma methods.

\section{Proposed methodology}

Applying lean and six-sigma methodologies have led to improvements in efficiency and satisfaction in different types of industries that provide products and/or services. Nevertheless, successful application of these approaches requires developing and following a systematic methodology. In this study, a practical methodology is proposed that can be followed and implemented to improve a specific aspect related to healthcare. The approach is divided into two main phases. Basic idea behind proposing this two-phase approach is to enhance the team/group development process.

In this type of study, the overall team/group usually comes from two different organizations. The first one is the group of people who perform the study and they are usually from outside the healthcare unit, while the second one is the group of people whom are working in the healthcare unit. It is well established that people forming a group to work as a team goes through different stages before they can work in harmony. According to Tuckman and Jensen (1977) ladder model, there are five stages for group development, which are forming, storming, norming, performing and adjourning. Since this type of study is usually performed within a limited time duration, it becomes vital that the group development process is accelerated. Hence, it is important to design a practical methodology that enhances team's development and minimizes any possible conflicts or misunderstanding. This is why the proposed approach consists of two main phases.

The first phase of the methodology is concerned with the activities performed a priori to the actual hospital visit. This phase aims to better prepare the team from outside the healthcare unit to identify the most promising areas of improvement as reported in literature and from meetings with experts from academia and healthcare sector. Furthermore, a set of possible questions is prepared and continuously reviewed such that this set of questions will further help the team to stay focus when performing the actual visits. Hence, the first phase enhances the understanding of the team members of what is needed and what is expected from them to achieve.

Once the outside team builds and acquires the required fundamentals, the second phase starts. The second phase is divided into three main steps, each step is concerned with a different but interrelated objective. In the first step of the second phase, the actual visit(s) to the concerned unit is performed. In this step, the team starts to be more familiar with the system/unit and its staff. In addition, they gradually start collecting answers for the questions they prepared. Once the team members are familiar with the unit and its staff, they start the second step of this phase, which is concerned about observation and data collecting process. This step requires the collaboration between the inside and outside groups of the team to be successful. This highlights why it is important to follow a methodology that accelerates the group development process. The third step in this phase is to start identifying the actual areas of improvements within the unit based on the observations and the collected data and then suggest the possible improvements accordingly. This step involves experts' opinion and feedback. The last two steps of the second phase is where tools 
and techniques that are part of for example lean and six-sigma methodology can be used as the team find adequate from the first step.

To have a better understanding of the proposed methodology, Figure 1 is developed with the objective to illustrate how this methodology is followed in the NICU case study. The details of the methodology are demonstrated in two phases as displayed in Figure 1. Basic steps that are followed to apply the proposed methodology on the NICU study are listed below:

- Literature review related to hospitals in general and NICU in particular from different countries.

- Preparation of questionnaires for the visits to the local hospital.

- Visits to the local hospital.

- Collection of responses from the prepared questionnaires and start to observe the process.

- Collection of data required to study the current staff and patient flow.

- Observation of the storage management and equipment.

- Observe, analyze and evaluation of the present staff/patients flow in terms of time and performance.

- Identification of the main areas of improvements in the unit.

- Suggested solutions and checking their validity and effectiveness.

- Improvement of the healthcare services and staff/patients flow.

The methodological steps are defined after extensive literature reviews and expert opinions from the NICU unit and academia. In addition, several brainstorming sessions between the authors and hospital authorities were arranged to define the procedural steps of the proposed methodology. Moreover, several visits to the hospital were conducted to get an initial idea about the NICU unit, followed by other visits with more specific objectives as discussed in the methodology. During these visits, the possible areas of improvement were noted down and important data and observations were recorded in order to have better understanding of the current practices and conditions at the unit. Furthermore, from these visits authors were able to modify the initially designed questionnaires and made them suitable to get intrinsic information about the NICU. The developed questionnaires were also iterated within the experts from the hospital to update and validate them in order to fulfill the study objectives. It is worth to point out that during these visits to the hospital, ethical issues, cultural and religion- beliefs, hospital's regulations and restrictions were discussed in details with the hospital representatives to ensure that there will be no violation of patients' privacy. The main privacy points are summarized in the following points:

- No violation of the privacy of the patients.

- Strictly, no photography is allowed without prior permission from patients and/or staff.

- The collected data and information are strictly used for the purpose of the study only.

- Not to publish patient information or any private information related to the unit or hospital in social media without prior permission.

- Results and findings should be presented anonymously. 


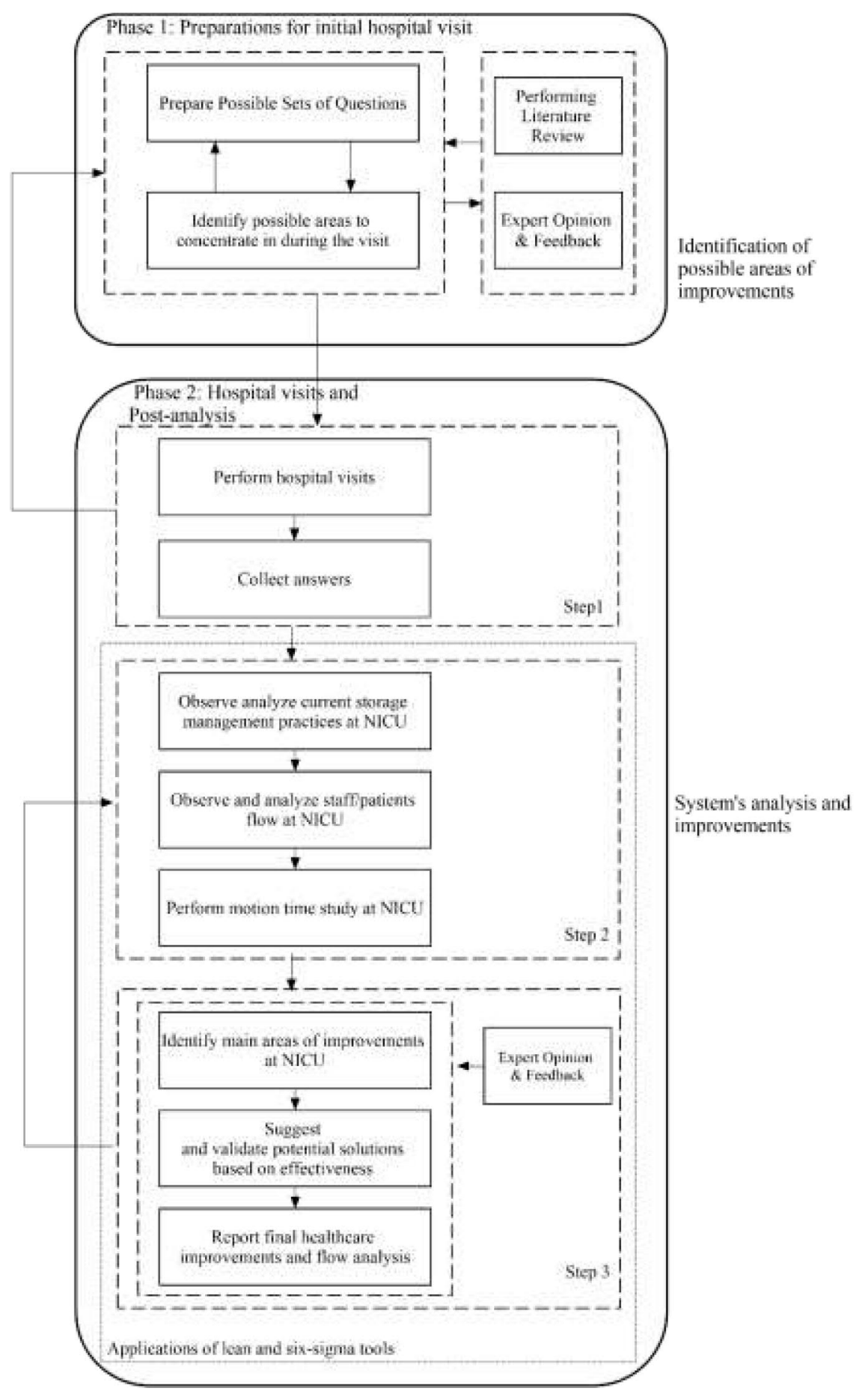

Figure 1. Proposed Methodology to study, analyze and improve healthcare services at NICU

\section{NICU case study description}

There are sixty-nine hospitals in Oman with a total capacity of more than 6400 beds. The current statistics shows that about $95 \%$ of the country's populations live within five miles of medical center (Oxford Business Group, 2020). . The studied hospital is considered as a major landmark in improvement of the healthcare service in the Sultanate of Oman. The case hospital is located at 
Muscat, Capital city of Oman. It provides a high level of healthcare for the patient with a maximum effectiveness, efficiency and patient's safety and satisfaction. In this study, the focus was mainly to improve a special unit in the hospital, which is known as Neonatal Intensive Care Unit (NICU). NICU is an intensive care unit, which is created and used for newborn babies that need special care and treatment. This unit may be known as Special Care Nursery, Intensive Care Nursery or Newborn Intensive Care Unit. The unit has flexibility to send and receive patients from other care units at different hospitals around the country.

Neonatal intensive care unit is divided into three major levels depending on the health conditions of the babies and their care needs. First level focuses on taking care of babies that are born with a low risk and with more stable conditions. Mainly premature babies born in 35 weeks and above are admitted into this level. Babies born in 32 weeks to 34 weeks are put in the second level as this level is dedicated to babies that are critically ill, requiring ventilator support, IV nutrition, and IV medications. If the baby's condition is worsen with time, he/she will be transferred to a higher NICU level. Third level provides care for infants weighing less than 1200 gm or having gestational maturity of less than 30 weeks. In addition, infants who have medical or surgical conditions, regardless of gestational age, should be cared for at the high dependency level (Floyd, 2020). Neonatal Intensive Care Unit is very important unit that needs to be ready for any new or urgent case. The surrounding environment must be accurately organized in terms of time, space, storage of necessary medical equipment and the movement of staff/patients within the unit.

\section{Data collection and results analysis}

The collected data are very helpful to achieve better understanding of the system, which helps in analyzing and suggesting possible ways to improve the efficiency of the NICU. As discussed in the methodology section, the data were collected by observing the staff flow, studying the working atmosphere and analyzing the storage management. Moreover, the team was involved in number of discussions with nurses and doctors working at the unit related to the nature of their works, the care provided to the patients and details of equipment in each store. To have more accurate and representative data about staff flow within the unit, the collection of flow data was performed at random days and timings for the three different NICU levels. In addition, the collected data from the stores were done with the cooperation of doctors and nurses. The data about noise level at the unit were collected using Sound Master Level phone application. The data collection process was done in random order to have details that reflect the usual normal conditions.

Each level of the NICU has different types of beds, such as incubators and coats. The square area of each bed is $80 \mathrm{~cm} \times 50 \mathrm{~cm}$, while the space between two beds is within the range of $60 \mathrm{~cm}$ to $90 \mathrm{~cm}$. Incubator is used for babies that are very small, considering their weights, ages and feeding processes. In each incubator, there is a drawer to keep patient's equipment (e.g. thermometer, extra sheets, bandages, cotton, etc.). Whereas, the coat is used for more stable babies. Table 2 shows the main tasks done by the staff at NICU and the average duration needed to complete the task.

Table 2. Main tasks done by the staff at NICU and average duration

\begin{tabular}{|l|c|l|}
\hline \multicolumn{1}{|c|}{ Task } & Average duration (min) & \multicolumn{1}{c|}{ Performed by } \\
\hline Blood collection & 7.25 & Doctor \\
\hline Discussing about patient condition & 7.78 & Doctor and nurse \\
\hline Checking ventilator records & 3 & Doctor \\
\hline
\end{tabular}




\begin{tabular}{|l|c|l|}
\hline Performing physical care needs & 6.4 & Nurse \\
\hline X-ray & 11 & Nurse and X-ray specialist \\
\hline Feeding & 8.25 & Nurse and mother \\
\hline Checking core temperature & 1 & Nurse \\
\hline
\end{tabular}

\subsection{Storage and corridor management}

There are six main stores located inside the NICU and a seventh store located outside of the unit. The team identified the equipment in each store and the frequency of usage of each one. The purpose behind this process is to determine the current arrangement of stores and looking for possible ways of improvement. We aimed to increase the storage capacity utilization in the best way that makes the access to equipment easier and make it faster to locate what the nurse is looking for. To achieve this, we implemented lean and Six-Sigma concepts to rearrange the stores in proper way. Nevertheless, we realized that due to limited storage capacity, the staff at the unit put number of equipment in the corridor. This resulted in impeding the movement of staff and patients. Table 3 shows the available machines, their usage frequencies and their quantity at the NICU.

Table 3. Machines, their usage frequency and quantity at NICU.

\begin{tabular}{|l|l|c|}
\hline Machine & Usage frequency & Quantity \\
\hline X- Ray & Very frequently used & 1 \\
\hline Ultrasonic & Very frequently used & 1 \\
\hline Phototherapy Capsule & Moderate frequently used & 2 \\
\hline Transport incubator & Occasionally used for within hospital transportation & 3 \\
\hline Transport incubator & Less frequently used for outside hospital transportation & 1 \\
\hline
\end{tabular}

From Table 3, it is seen that some of the machines are more frequently used than others. However, putting all of them in the corridor (as currently practiced) resulted in impeding the staff movement and narrowing the corridor.

\subsection{Noise level}

Hospital environment is a busy and noisy place by nature due to the interactions between staff, patients, visitors and the continuous usage of machines and equipment. The noise may affect the heeling process and may cause discomfort for the patients (Standley, 2012). It may also affect the performance of the staff, as noisy environments may reduce the level of concentration. Hence, it is crucial to provide a suitable environment especially at the NICU that takes care of the premature infants. Based on a report from the US Environmental Protection Agency (EPA), the noise level at NICU should be lower than $45 \mathrm{~dB}$. The exposure to noise above $45 \mathrm{~dB}$ may result in cochlear damage or disrupt the normal growth and development of premature infants (American Academy of Pediatrics, 1997). The noise level at NICU was measured by using phone application called Sound Master Level at different days and times. Table 4 summarizes the recorded noise levels in different days.

Table 4. Noise Level Recorded By Sound Master App at NICU Levels

\begin{tabular}{|l|c|}
\hline \multicolumn{1}{|c|}{ Date } & Noise Level $(d B)$ \\
\hline $29-10-2015$ & 79 \\
\hline $10-11-2015$ & 58 \\
\hline
\end{tabular}




\begin{tabular}{|l|l|}
\hline $27-11-2015$ & 72 \\
\hline $2-12-2015$ & 51 \\
\hline Average Noise Level & 65 \\
\hline
\end{tabular}

According to OSHA standard, the noise level at daytime should be around $50 \mathrm{~dB}$ and at nighttime around $45 \mathrm{~dB}$. However, the recorded noise level at the unit is always above the standard. All records were measured at daytime and the average was calculated to be $65 \mathrm{~dB}$, which is more than the standard level by $15 \mathrm{~dB}$. The nurses at the unit confirmed that at night the noise level is above the standard levels as well. Therefore, it is necessary to focus on this high noise level as a major source of discomfort.

\section{Suggested improvements to the NICU}

From the collected data and observations, it was revealed that NICU has number of drawbacks that need to be looked at. In order to suggest suitable solutions, these drawbacks were carefully studied and analyzed. During this process, experts were consulted as suggested in the methodology section. This section highlights the suggested improvements for the three main identified drawbacks at the NICU, which are the staff flow at NICU, storage management and noise level.

\subsection{The staff flows at NICU}

The flow of nurses and doctors at NICU to perform different activities was observed and time was recorded and presented in Table 2. After analyzing the data in Table 2, it is observed that the arrangement of tables, beds, computers, tools and equipment affects the flow of nurses and doctors inside the unit. Hence, the flow time can be reduced in order to increase the efficiency at the unit. Time and motion study analysis is one way that can be used to study the flow of different things at the unit. It was clear that time and motion study analysis performed at the unit would enable easier flow and hence reduce the flow time. This makes the place more comfortable. For the low dependency level room, it was found that the current arrangement of that room is efficient. However, our analysis suggested that modifications to the current layout in the other two rooms by rearranging few things would help in smoothening the flow. For example, from Figure 2(a), it evident if the table at the middle of the room is removed, it will give high accessibility to reach any bed in the room without any disturbance as seen in Figure 2(b). Following are specific suggestions given to improve the flow at the high and at the intermediate dependency levels as both have similar layouts.

- Move the two computers to the sidewall and put them on shelves such that staff can use them while standing. This minimizes the space occupied by the computers and eliminates the need for chairs.

- For the frequently needed consumables, we suggested to add a vertical cupboard with shelves and put them on the sidewall as shown in Figure 2(b). This can be used to also store small equipment. This enhances the efficiency of the work and reduces the travelling time of nurse to bring some frequently used consumable or small equipment while not obstructing the motion within the room.

- This new re-arrangement as shown in Figure 2(b) resulted in the possibility of having a new passage between the two sides of the room that can be used in normal situations by staff to move more easily.

- In case of emergency when more beds are needed, the new arrangement provides more available space to add two extra beds, which increases the space utilization. 


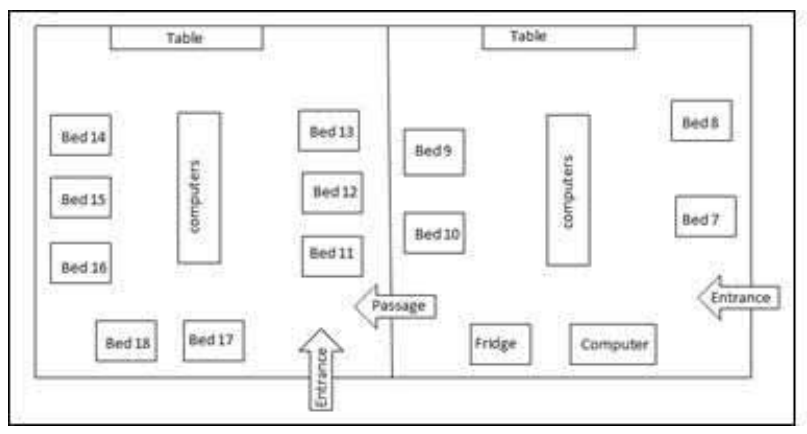

(a)

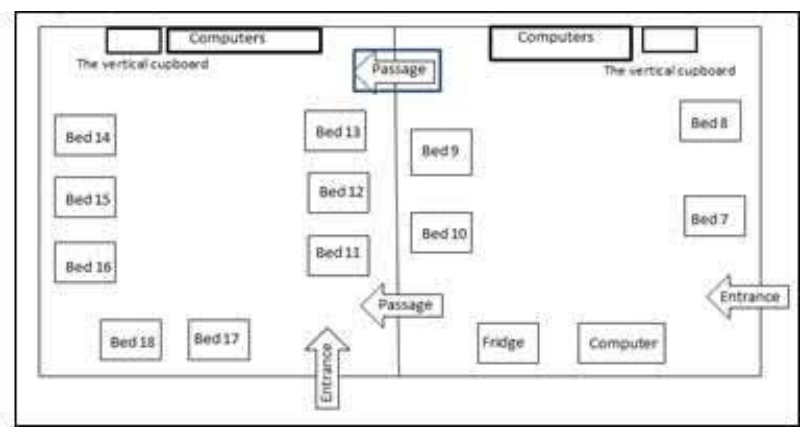

(b)

Figure 2. High/Intermediate Dependency Rooms Layout (a) before and (b) after the Suggested Improvements

\subsection{Storage management}

Healthcare services at this NICU are required to be on time and without delays while ensuring to provide the right care to the right patient. Hence, all staff movements at the unit are critical as they directly affect the service efficiency. Therefore, managing the NICU stores to fully utilize their capacity in an efficient way will have a positive impact on the overall performance. This is due to the expected increase in the accessibility of the stores, the reduced time needed to search for and reach items and the increase in the stores' capacity utilization, as space is always a major concern. After studying and analyzing the different stores of the unit, concepts of lean, six-sigma and relayout were carefully applied to the medical stores. Based on the discussions with the unit's authorities in the hospital, it was agreed that the team will be allowed to examine and suggests improvements to three stores. The final suggestions for improvements of these four stores are stated below:

\section{Store 1: General store}

The main items stored at this store are oxygen cylinders of two types, light oxygen cylinder and heavy oxygen cylinder; suctions, infusion pumps, and oxygen analyzer. Following bullets summarizes suggested areas of improvement to this store:

- First, renaming the store from general store to equipment store. The suggested name is better consistent with items it stores. This will make it easier to locate the store and its content. This will also help new staff, vendors, maintenance teams, auditors, etc. to recognize this store.

- Second, providing a detailed list of all items that are kept in this store. This list should be hanged on the door of the store. As suggested, this step may affect the performance of the staff and vendor administrator to know the items stored inside it. This saves the time spent in searching for a specific item.

- Third, the store contains $\mathrm{O}_{2}$ cylinders that have to be stored according to the HTMO2 guidelines. Hence, a brief summary of these guidelines should be hanged next to the stored cylinders as a reminder of the proper handling procedures.

- Finally, adding colored signs/boards for each shelf that indicates the item name and quantity that should be placed on it. This will make it easier to organize the store shelves and may help the staff to reach the required item by recognizing the board color. It will also make it easier for the vendor administrator to check the items and their availability. 
Figure 3 shows the current store and beside it a sketch of some of suggested improvements.

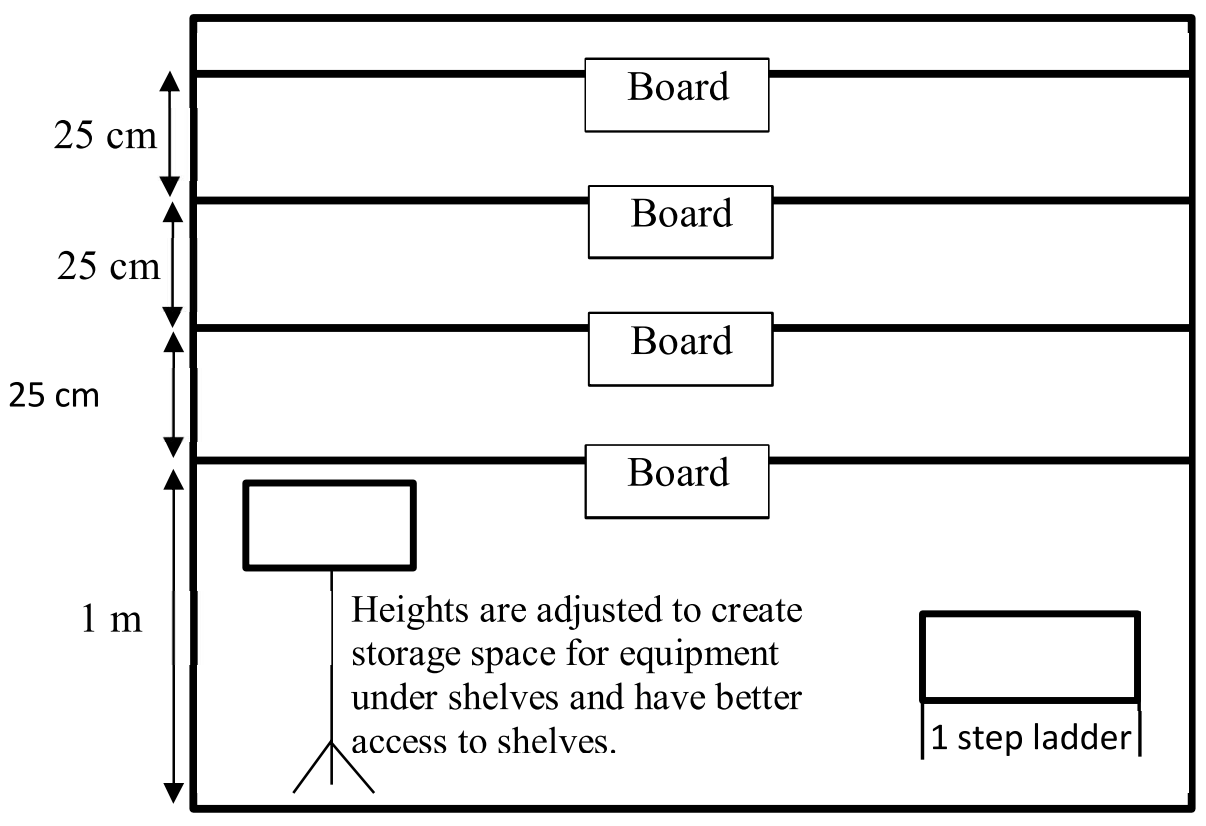

Figure 3. Sketch of the Suggested Improvement in the Equipment Store

Store 2: Respiratory store

The respiratory store should contain all items and machines that are related to respiratory such as ventilators. There are many suggestions to improve the store utilization. One of the suggestions is providing a list of items that should be placed inside the store. The benefits of this step are as discussed previously. Further, the store contains colored boards tagging the item's name. However, all boards are red and the font size is small. Therefore, varying the boards' colors and write the name of item in a larger font size will enhance the store's management. This will help to better organize the store, and improve staff attitude towards organization. The current design of the store has short shelves. Hence, extending the length of these shelves will increase the capacity of the store. In addition, there is a sink kept in the store, which needs to be disposed, as it is not in use. Furthermore, there are three types of respiratory equipment. This equipment has approximately the same dimensions and shape ( $1 \mathrm{~m}$ long with wheels). From Figure 4, it is seen that all the three equipment are placed at the middle of the store, which makes it difficult to reach the shelves. Every time a person needs to reach something from the shelves, he/she has to move the equipment to get an access to the shelves. To improve the current situation, adding short partitions to create area for each type of the three equipment can improve the situation. Left sketch of Figure 4 shows the proposed partitions and other modifications that may be applied to improve the storage capacity. 


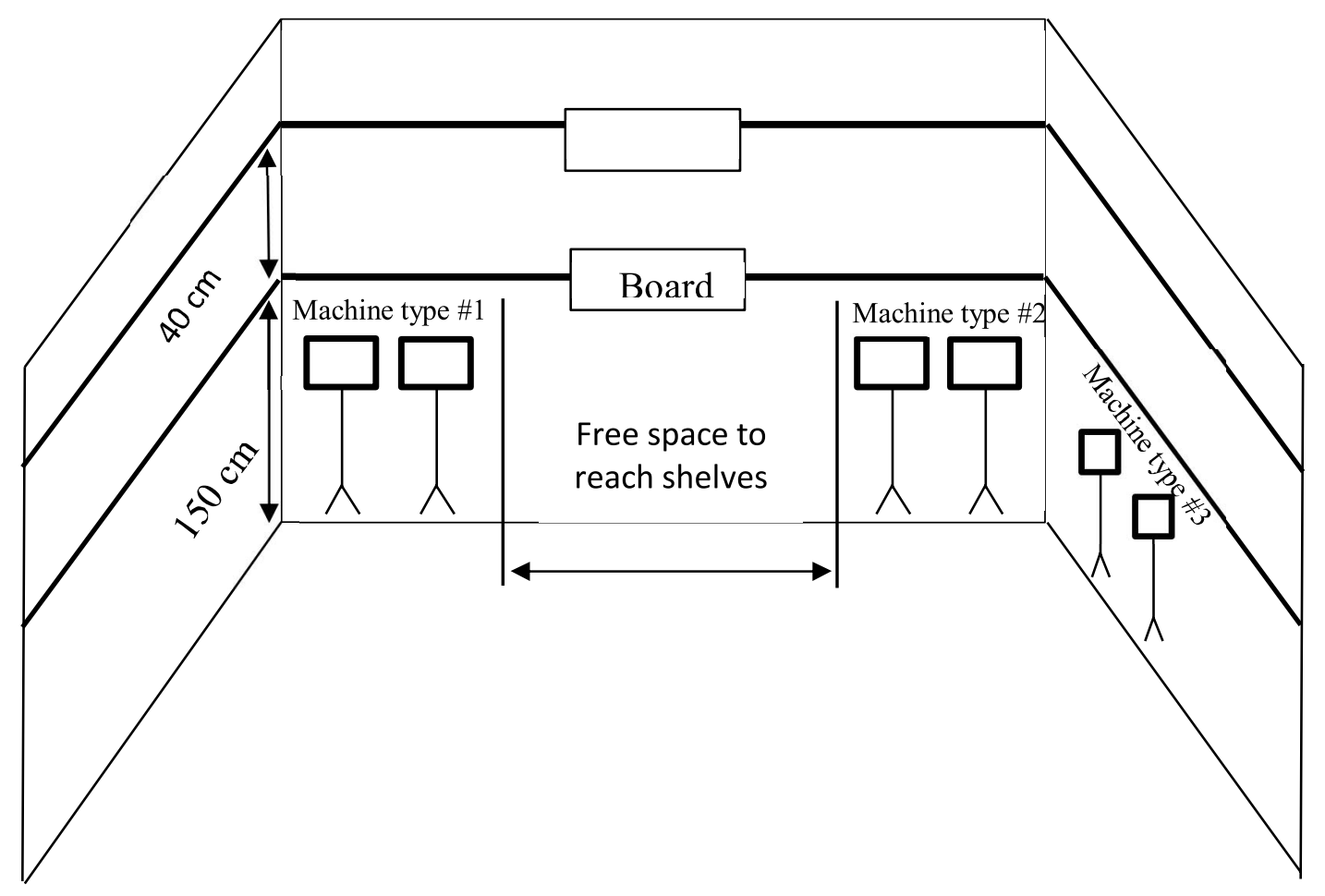

Figure 4. Sketch of the Suggested Improvement in the Respiratory Store

Store 3: Clean utility store

Doctors and nurses use this store frequently at the same time. The store width is $220 \mathrm{~cm}$ and its length is about $340 \mathrm{~cm}$. It contains medicines, files, a fridge and arterial-blood gas (ABG) test machine. The current arrangement is proper. However, the main concern is complains from medical team members about the need to frequently use the ABG machine which causes congestions. Hence, the study team decided to explore the motion of the doctors and nurse within the unit relevant to the usage of this testing machine. This helps to analyze the current situation. The team adapted the use of spaghetti diagram to observe the current flow of the nurses and doctors. A spaghetti diagram is a visual representation using a continuous flow line tracing the path of an item or activity through a process. Another benefit from this spaghetti diagram is to highlight the major intersection points within the unit. Areas where many motion lines overlap causes delay. The developed Spaghetti diagram is shown in Figure 5, where blue lines represents the doctors' flow and the black lines represents the nurses' flow. Having more motion lines within a certain region indicates that this is a more critical area. 


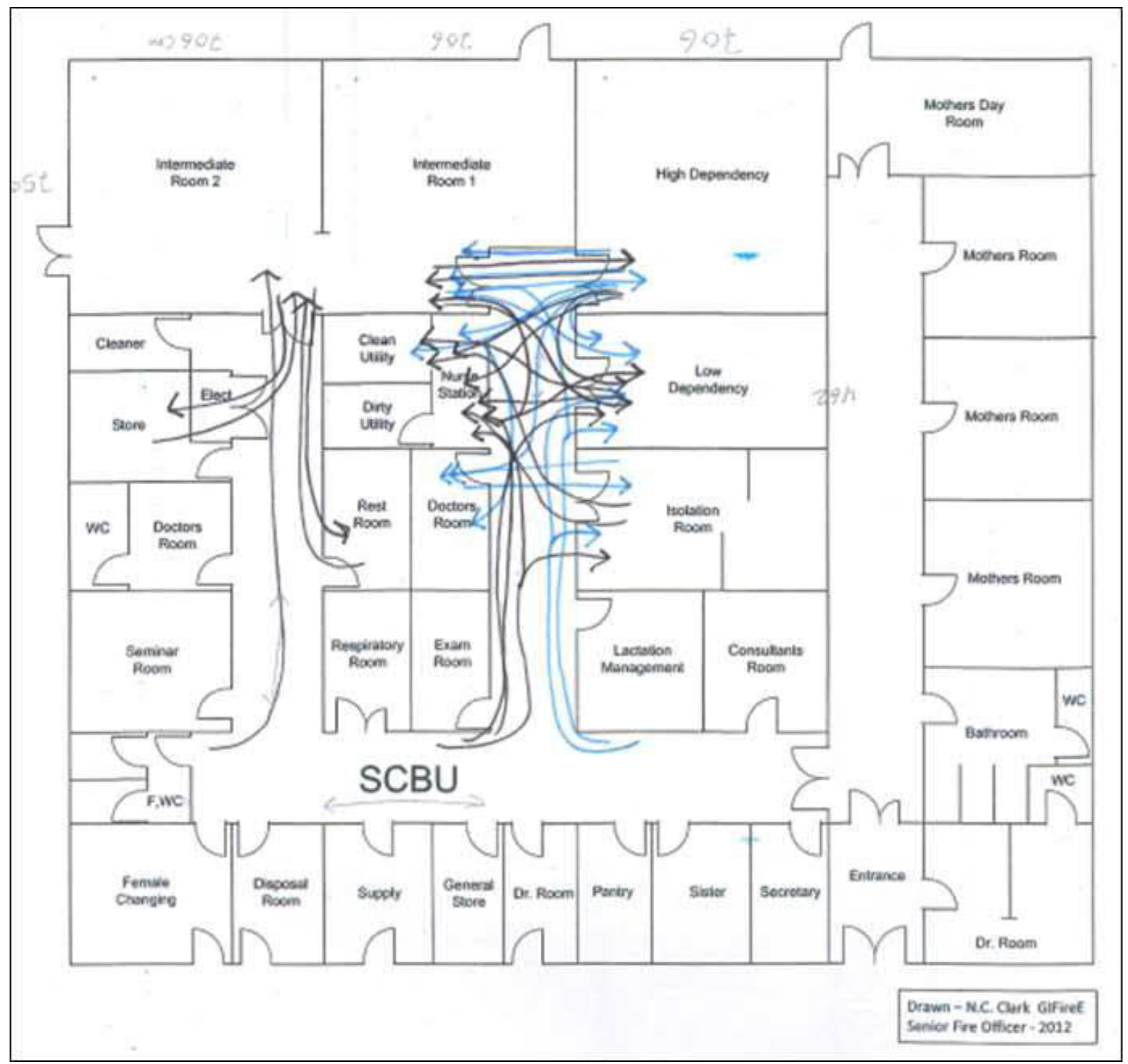

Figure 5. Spaghetti Diagram

From Figure 5, it is revealed that the store and the area around it is a critical area for the process. However, the frequency of entering that store is due to the usage of the ABG machine by the doctors. Further, the corridor between the high and intermediate dependency room has high motion level, which is partially attributed to the need of using the testing machine. Hence, as an alternative approach to improve the current situation and ease the congestion within that area, it is suggested to move the ABG from the store to the intermediate dependency room. This suggestion is actually possible as a part of the suggestions discussed in subsection 6.1 and the saved space as shown in Figure 2.

\subsection{Noise level}

Noise level at NICU plays a major role in the communication of staff, interactions of families, and development of infant. As recorded in Table 4, the noise level is above the OSHA standards. From 
this perspective, it is necessary to focus on this issue and suggested possible solutions to reduce the noise level. Following are some of the suggested techniques that can be helpful in reducing the noise level to the standard or near standard level at NICU.

\subsubsection{HUSH policy}

Majority of the patients complains about the noises around them, which are not permitting them to have enough sleep. This effects their mood and recovery process. Hence, noise can be considered as a direct and indirect obstacle for the recovery process. The sources of such noises are from interaction between the staff, patients families, visitors, workers, etc., as well as from medical machineries and equipment. Noises due to machines and equipment may be inevitable, as some of these are actually needed for monitoring purposes. However, noises due to human interaction can be controlled. During the data collection, the study team observed that the staff at the unit keeps talking about unnecessary things or things that are unrelated to the patients' care, which is very disturbing for patients.

A possible approach to overcome such noises due to unnecessary talking can be controlled by applying what is known as the HUSH (Help Us Support Healing) policy at NICU and encouraging staff/people to adopt it. In fact, the noise level while talking is directly proportional to the surrounding noise level. People tend to raise their voices when the surrounding place is noisy. Hence, the HUSH policy is very simple policy that keeps reminding the staff/people to lower their voices and not to talk unless it is required.

\subsubsection{Acoustic panels}

While the suggested HUSH policy in the previous subsection may be an adequate first step to enhance people awareness, however, another approach is needed to deal with sounds coming from medical devices. It has been proved that adding furniture with fabric is an easy way to dampen sound within a room. The main reason behind this is that this type of furniture has the ability to absorb sound waves. Nevertheless, due to space constraints, the furniture added to the medical unit must not consume space. Hence, the best type of this furniture is what is known as acoustic panels. They can be easily hanged on walls to create different childish designs that enhance the general atmosphere within the unit. The backside of these panels is made of foam and is covered by fabric. This design gives them the ability to absorb the noises. Moreover, having these acoustic panels at different locations may have the advantage of using them as a pin board such that staff can post papers or notes on them.

\section{Conclusions}

Improving healthcare services is very important to maintain and achieve the high-level health standards. However, healthcare system is a very complicated system that can be affected by different factors. Hence, it is essential to identify these factors and understand relative relationship between the factors and their impacts on each other. One important side that can enhance healthcare services is the working environment and its surroundings. Therefore, this research study proposed an innovative methodology that can be followed to improve a healthcare unit. The methodology is built with emphasis to accelerate team development process and draw a clear roadmap on how to select and use proper tools. The proposed methodology helps to determine the appropriate ways to identify the possible areas of improvement within a unit based on collected data and observations. This strategy aids to clarify the nature of the concepts and methods that can be implemented to achieve an efficient healthcare system. 
To illustrate the efficacy of the proposed methodology, it was applied to a neonatal intensive care unit (NICU) in a hospital in Oman with the aim to improve the work environment. Following the proposed methodology, the study team found many opportunities for potential improvements in the unit. These improvements will have a positive impact to efficiently improve the staff flow and storage management and reducing the noise level. Furthermore, collected feedback from the staff of the unit revealed that they enjoyed working with the outside group. The staff felt more comfortable talking and dealing with the outside group, as the outside group showed a high level of credibility in understanding the nature of the NICU work and many of the technical terminologies used by them. This demonstrates how successful the proposed methodology was to accelerate the team development process.

\section{References}

American Academy of Pediatrics. (1997), "Noise: A hazard for the fetus and newborn", Pediatrics, Vol. 100 No. 4, pp. 724-727.

Antony, J., Palsuk, P., Gupta, S., Mishra, D. and Barach, P. (2018), "Six Sigma in healthcare: a systematic review of the literature", International Journal of Quality \& Reliability Management, Vol. 35 No. 5, pp. 1075-1092.

Benzies, K.M., Shah, V., Aziz, K., Lodha, A. and Misfeldt, R. (2019), "The health care system is making 'too much noise' to provide family-centred care in neonatal intensive care units: perspectives of health care providers and hospital administrators", Intensive and Critical Care Nursing, Vol. 50, pp. 44-53.

Butler, M., Szwejczewski, M. and Sweeney, M. (2018), "A model of continuous improvement programme management", Production Planning \& Control, Vol. 29 No. 5, pp. 386-402.

Chalmers, B. (2017), "Family-centred maternity and newborn care in Canada: underlying philosophy and principles", Public Health Agency of Canada(Ed.), Family-Centred Maternity and Newborn Care: National Guidelines, Government of Canada, Ottawa, ON.

Costa, L.B.M., Rentes, A.F., Bertani, T.M. and Mardegan, R. (2017), "Lean healthcare in developing countries: Evidence from Brazilian hospitals", The International Journal of Health Planning and Management, Vol. 32 No. 1, pp. e99-e120.

Costeloe, K., Hennessy, E., Stacey, F., et al. (2009), "Survival of extremely preterm infants by hospital designation", Acta Paediatrica, 98 (Suppl 460).

DelliFraine, J.L., Langabeer, J.R. and Nembhard, I.M. (2010), "Assessing the evidence of six sigma and lean in the health care industry", Quality Management in Health Care, Vol. 19 No. 3, pp. 211-225.

Floyd (2020), available at: https://www.floyd.org/medicalservices/maternity/NICU/Pages/Levels-of-Neonatal-Care.aspx, accessed at 30 June 2020.

Henrique, D.B. and Filho, M.G. (2020), "A systematic literature review of empirical research in Lean and Six Sigma in healthcare", Total Quality Management \& Business Excellence, Vol. 31 N0. 3-4, pp. 429-449, published online: 30 Jan 2018.

Improta, G., Balato, G., Ricciardi, C., Russo, M.A., Santalucia, I., Triassi, M. and Cesarelli, M. (2019), "Lean Six Sigma in healthcare: Fast track surgery for patients undergoing prosthetic hip replacement surgery", The TQM Journal, Vol. 31 No. 4, pp. 526-540.

Jochen, P., Paul J.S., Xin, C., Courtney, C.N., Eric, J.T., Daniel, S.T., Henry, C.L., David, D. and Bryan, S.J. (2018), "The correlation between neonatal intensive care unit safety culture and quality of care", Journal of Patient Safety, November 7, Volume Publish Ahead of Print. 
Joseph, K.S., Kramer, M.S., Allen, A.C., Cyr, M., Fair, M., Ohlsson, A. and Wen, S.W. (2000), "Gestational age- and birthweight-specific declines in infant mortality in Canada, 1985-94", Pediatric and Perinatal Epidemiology, Vol. 14, No. 4, pp. 332-339.

Lakshmanan, A., Agni, M., Lieu, T., Fleegler, E., Kipke, M., Friedlich, P.S., McCormick, M.C. and Belfort, M.B. (2017), "The impact of preterm birth $<37$ weeks on parents and families: a cross-sectional study in the 2 years after discharge from the neonatal intensive care unit", Health and Quality of Life Outcomes, Vol. 15, Article number: 38.

Loay, S. and DeYoung, C. (2003), "Six Sigma in health care", International Journal of Health Care Quality Assurance, Vol.16, No. 4, 2003, pp. 1-5.

McGowan, E.C., DuBS, N. Hawes, K., Tucker, R., O'Donnell, M. and Vohr, B. (2017), "Maternal mental health and neonatal intensive care unit discharge readiness in mothers of preterm infants", The Journal of Pediatrics, Vol. 184, pp. 68-74.

Moore, T., Hennessy, E. M., Myles, J., Johnson, S. J., Draper, E. S., Costeloe, K. L., \& Marlow, N. (2012). Neurological and developmental outcome in extremely preterm children born in England in 1995 and 2006: the EPICure studies. Bmj, 345, e7961.

Oxford Business Group (2020), Oman's health care system increasingly ready to meet the population's needs, Available at: https://oxfordbusinessgroup.com/overview/rise-growingpopulation-finds-health-care-system-ready-meet-its-needs, accessed on 21 October 2020.

Pande, P.S., Neuman, R.P. and Cavanagh, R.R. (2001), The Six Sigma Way: How GE, Motorola, and Other Top Companies are Honing Their Performance. New York, NY: McGraw-Hill Professional. ISBN 0-07-135806-4, OCLC 647006794, Pande Six Sigma Way.

Patel, R.M., Kandefer, S., Walsh, M.C., Bell, E.F., Carlo, W.A., Laptook, A.R., Sánchez, P.J., Shankaran, S., Van Meurs, K.P., Ball, M.B. and Hale, E.C., 2015. Causes and timing of death in extremely premature infants from 2000 through 2011. New England Journal of Medicine, Vol. 372 No. 4, pp.331-340.

Peimbert-García, R. E. (2019). Analysis and Evaluation of Reviews on Lean and Six Sigma in Health Care. Quality Management in Healthcare, Vol. 28No. 4, 229-236.

Rodrigo, P-G. (2019), "Analysis and evaluation of reviews on lean and six sigma in health care", Quality Management in Health Care, Vol. 28 No. 4, pp. 229-236.

Standley, J. (2012), "Music therapy research in the NICU: an updated meta-analysis, Neonatal Network: The Journal of Neonatal Nursing, pp. 311-316.

Stelson, P., Hille, J., Eseonu, C. and Doolen, T. (2017), "What drives continuous improvement project success in healthcare?", International Journal of Health Care Quality Assurance, Vol. 30 No. 1, pp. 43-57.

Sunder, M.V., Ganesh, L.S. and Marathe, R.R. (2018), "A morphological analysis of research literature on lean six sigma for services", International Journal of Operations \& Production Management, Vol. 38 No. 1, pp. 149-182.

Sunder, M.V. and Kunnath, N.R. (2020), "Six Sigma to reduce claims processing errors in a healthcare payer firm", Production Planning \& Control, Vol. 31 No. 6, pp. 496-511.

Trajkovski, S., Schmied, V., Vickers, M.H. and Jackson, D. (2016), "Experiences of neonatal nurses and parents working collaboratively to enhance family centred care: the destiny phase of an appreciative inquiry project", Collegian, Vol. 23, pp. 265-273.

Tuckman, B. W. and Jensen, M. C. (1977), "Stages of Small Group Development Revisited", Group and Organizational Studies, Vol. 2, pp. 419-427 
Vaishnavi, V. and Suresh, M. (2020), "Assessment of readiness level for implementing lean six sigma in healthcare organization using fuzzy logic approach", International Journal of Lean Six Sigma, Vol. ahead-of-print No. ahead-of-print, (DOI: 10.1108/IJLSS-07-2019-0081)

Webmd (2020), Available at: https://www.webmd.com/baby/guide/premature-labor\#1, accessed on 23 June 2020. 